\title{
Biologika im Langzeitvergleich
}

Bei Psoriasis-Patienten hat die Behandlung mit Ustekinumab im Vergleich zu Etanercept die höchste Fünfjahreseffektivität. Zudem ist nach einem Jahr die Wahrscheinlichkeit für ein PASI-75-Ansprechen durch eine Therapie mit Adalimumab oder Ustekinumab höher als mit Etanercept.

Zur Beurteilung der bei Psoriasis am häufigsten verschriebenen Biologika, werteten Dermatologen um Dr. Jeffrey Zweegers* von der Radboud-Universität in Nijmegen in den Niederlanden Daten aus der klinischen Praxis aus, die seit 2005 im BioCAPTURE-Register (Continuous Assessment of Psoriasis Treatment Use Registry with Biologics) dokumentiert werden. Die Daten wurden zu Beginn des Studienzeitraums erhoben sowie sechs und zwölf Wochen später, dann alle drei Monate für ein Jahr und danach alle drei bis sechs Monate.

Der Erfolg der jeweiligen Behandlung wurde anhand des Verlaufs der PASI-Werte bzw. anhand der erreichten PASI-75-Reduktion im Vergleich zum Studienbeginn beurteilt. Zweegers und seine Kollegen erfassten das Ansprechen auf die Behandlung mittels "multilevel linear regression analyses" (MLRA) und "generalized estimating equation analyses" (GEE). Dabei erfolgt u.a. eine Mehrebenenregressionsanalyse, bei der berücksichtigt wird, dass bei einem Patienten mehrere Therapieepisoden vorkommen. Darüber hinaus wurden die Ergebnisse um Störfaktoren bereinigt, etwa Alter, Geschlecht, positive Familienanamnese, Rauchen sowie frühere Behandlungen mit Biologika.

Für die MLRA-Berechnungen wurden 513 Therapieepisoden bei $356 \mathrm{~Pa}$ tienten berücksichtigt. 178 Therapieepisoden erfolgten mit Adalimumab, 245 mit Etanercept und 90 mit Ustekinumab. In die GEE-Analyse wurden 483 Therapieepisoden einbezogen. Im Median lag der PASI zu Studienbeginn bei 13,1 , also ähnlich hoch wie in anderen Registern für Psoriasis-Patienten.
DiehöchsteTherapieeffektivität über fünf Jahre stellten die Ärzte bei Behandlung mit Ustekinumab fest. Mithilfe der GEE-Analyse ermittelten sie für Adalimumab und Ustekinumab eine größere Wahrscheinlichkeit, nach einem Jahr Behandlung ein PASI-75-Ansprechen zu erzielen als mit Etanercept. Und: Eine höhere Dosierung als empfohlen wurde sowohl nach einem Jahr als auch nach fünf Jahren bei mehr Patienten verabreicht, die Etanercept erhielten, als bei Patienten, die mit den anderen beiden Biologika therapiert wurden. Das war nach einem Jahr mit Adalimumab bei $31,5 \%$, mit Etanercept bei $55,1 \%$ und mit Ustekinumab bei nur 17\% der Patienten der Fall. Nach fünf Jahren lag der Anteil ähnlich hoch: mit Adalimumab bei 39,3\%, mit Etanercept bei $71,4 \%$ und mit Ustekinumab bei $24 \%$. Das entspricht auch den Beobachtungen in ihrer systematischen Übersicht, wie die Ärzte berichten. Die Unterschiede zwischen den Gruppen sind signifikant. Die therapeutische Wirksamkeit höherer Dosen der Biologika sei über den gesamten Studienzeitraum ähnlich gewesen, so Zweegers und seine Kollegen. Die Studienergebnisse müssten jedoch noch in anderen prospektiven Studien zur Anwendung der Präparate in der täglichen Praxis bestätigt werden.

\footnotetext{
* Die Autoren weisen darauf hin, dass die University Medical Center St. Radboud Foundation das Projekt finanziert hat und dafür von den Unternehmen Pfizer, Janssen und AbbVie unterstützt worden ist.
}

Bericht: Peter Leiner, springermedizin.de

hautnah $2017 \cdot 16: 45$

DOI 10.1007/s12326-017-0238-4

C) Springer-Verlag Wien 2017
Hier steht eine Anzeige. Springer 